\title{
Hepatic blood flow distribution and performance in conventional and novel Y-graft Fontan geometries: A case series computational fluid dynamics study
}

\author{
Weiguang Yang, MS, ${ }^{\mathrm{a}}$ Irene E. Vignon-Clementel, $\mathrm{PhD},{ }^{\mathrm{b}}$ Guillaume Troianowski, MS, ${ }^{\mathrm{c}}$ \\ V. Mohan Reddy, MD, ${ }^{d}$ Jeffrey A. Feinstein, MD, MPH, ${ }^{\mathrm{e}}$ and Alison L. Marsden, $\mathrm{PhD}^{\mathrm{a}}$
}

\begin{abstract}
Objectives: A novel Y-shaped baffle has been proposed for the Fontan operation with promising initial results. However, previous studies have relied either on idealized models or a single patient-specific model. The objective of this study is to comprehensively compare the hemodynamic performance and hepatic blood flow distribution of the Y-graft Fontan baffle with 2 current designs using multiple patient-specific models.
\end{abstract}

\begin{abstract}
Methods: Y-shaped and tube-shaped grafts were virtually implanted into 5 patient-specific Glenn models forming 3 types of Fontan geometries: Y-graft, T-junction, and offset. Unsteady flow simulations were performed at rest and at varying exercise conditions. The hepatic flow distribution between the right and left lungs was carefully quantified using a particle tracking method. Other physiologically relevant parameters such as energy dissipation, superior vena cava pressure, and wall shear stress were evaluated.
\end{abstract}

Results: The Fontan geometry significantly influences the hepatic flow distribution. The Y-graft design improves the hepatic flow distribution effectively in 4 of 5 patients, whereas the T-junction and offset designs may skew as much as $97 \%$ of hepatic flow to 1 lung in 2 cases. Sensitivity studies show that changes in pulmonary flow split can affect the hepatic flow distribution dramatically but that some Y-graft and T-junction designs are relatively less sensitive than offset designs. The Y-graft design offers moderate improvements over the traditional designs in power loss and superior vena cava pressure in all patients.

Conclusions: The Y-graft Fontan design achieves overall superior hemodynamic performance compared with traditional designs. However, the results emphasize that no one-size-fits-all solution is available that will universally benefit all patients and that designs should be customized for individual patients before clinical application.

(J Thorac Cardiovasc Surg 2012;143:1086-97)

The impact of geometry on Fontan hemodynamics is now widely accepted in the engineering and clinical communities. ${ }^{1-4}$ Recent advances in computational fluid dynamics, computer-aided design, magnetic resonance imaging (MRI), and fluid structure interaction methods for blood flow modeling have led to studies of Fontan hemodynamics and surgical design progressing from idealized to patientspecific models, steady to unsteady flow, and trial and error to optimal design. ${ }^{5-9}$

From the Department of Mechanical and Aerospace Engineering, ${ }^{\text {a }}$ University of California San Diego, La Jolla, Calif; INRIA Paris-Rocquencourt, ${ }^{\mathrm{b}}$ Le Chesnay Cedex, France; and Institute for Computational and Mathematical Engineering, ${ }^{c}$ Department of Cardiothoracic Surgery, ${ }^{\mathrm{d}}$ and Departments of Pediatrics and Bioengineering, ${ }^{\text {e }}$ Stanford University, Stanford, Calif.

This work was supported by the American Heart Association, a Burroughs Wellcome Fund Career Award at the Scientific Interface, a Leducq Foundation Network of Excellence grant, and INRIA associated team grant.

Disclosures: Authors have nothing to disclose with regard to commercial support.

Received for publication Dec 1, 2010; revisions received May 13, 2011; accepted for publication June 27, 2011; available ahead of print Sept 12, 2011.

Address for reprints: Alison L. Marsden, MD, Department of Mechanical and Aerospace Engineering, University of California San Diego, 9500 Gilman Dr, La Jolla, CA 92093-0411 (E-mail: amarsden@ucsd.edu).

$0022-5223 / \$ 36.00$

Copyright (c) 2012 by The American Association for Thoracic Surgery doi:10.1016/j.jtcvs.2011.06.042
In the earlist extracardiac Fontan procedures, the inferior vena cava (IVC) was anastomosed to the pulmonary arteries (PAs) via a straight polytetrafluoroethylene graft (Gore-Tex graft; W. L. Gore \& Associates, Inc, Flagstaff, Ariz) forming a T-junction. An offset design derived from simulations ${ }^{5}$ was then adopted by surgeons to reduce energy losses and remains standard of care in most extracardiac procedures.

Recently, 2 research groups ${ }^{7,10}$ proposed a new Y-shaped connection to replace current designs. First, Soerensen and coworkers ${ }^{10}$ bifurcated both the superior vena cava (SVC) and IVC. Simulations using an idealized model with steady inflow conditions demonstrated lower energy loss. Given the technical difficulties and need for increased synthetic material, this modification was not pursued in clinical practice. Marsden and coworkers ${ }^{7}$ introduced an IVC-only bifurcated Fontan modification. A detailed comparison of energy efficiency, Fontan pressures, and hepatic flow distribution demonstrated superior Y-graft performance. ${ }^{7}$ An optimization process was then used to systematically explore design parameters and hemodynamics for an idealized Y-graft model. ${ }^{8}$ Although the preliminary studies showed the Y-graft design to improve hemodynamics overall, it has not yet been confirmed that the superiority of the Y-graft is universal. 

Abbreviations and Acronyms
IVC = inferior vena cava
LPA $=$ left pulmonary artery
MRI = magnetic resonance imaging
$\mathrm{PA}=$ pulmonary artery
$\mathrm{RPA}=$ right pulmonary artery
$\mathrm{SVC}=$ superior vena cava
$\mathrm{WSS}=$ wall shear stress

Complications after the Fontan procedure, including diminished exercise capacity, thromboembolic complications, protein-losing enteropathy, arteriovenous malformations, ventricular dysfunction, and arrhythmias, continue to present serious clinical challenges despite postoperative survivals upwards of $90 \% .^{11}$ Among these, the development of pulmonary arteriovenous malformations is known to be linked to hepatic flow distribution and is therefore an obvious target for improvement via simulation-based design. Although the pathogenesis of pulmonary arteriovenous malformations is unknown, several studies have revealed a clear link between the absence of hepatic venous return and the development of pulmonary arteriovenous malformations, and reversal of pulmonary arteriovenous malformations has been achieved by correcting the uneven hepatic flow distribution. ${ }^{12}$ Although the energy dissipation of different Fontan geometries has been investigated in numerous studies ${ }^{1-3,5,6}$ only a few studies ${ }^{4,7,13-16}$ have quantified the hepatic flow distribution in in vitro or simulation studies.

The purpose of this article is to evaluate the potential performance of the Y-graft Fontan procedure in simulation as a step toward clinical implementation. Multiple virtual patient models are used, and multiple parameters ${ }^{4}$ are evaluated for each, with particular attention paid to the hepatic flow distribution.

\section{METHODS}

\section{Geometric Model Construction}

Virtual surgery was performed on 5 models by implanting a Y- or tubeshaped graft into patient-specific Glenn models ${ }^{17}$ constructed from MRIs. All patients gave consent as part of an institutional review board approved protocol at Lucile Packard Children's Hospital (Stanford University). Model construction was performed using a custom version of the open source Simvascular software package, ${ }^{18}$ as in our previous work. ${ }^{7}$

Geometric parameters were chosen on the basis of our previous optimization study. ${ }^{8}$ A Y-graft with a 20 -mm diameter trunk and 15 -mm diameter branches was chosen for all patients. The T-junction and offset models were constructed with a 20-mm diameter tube-shaped graft following common clinical practice, and resulting models are shown in Figure 1.

In the virtual implantation, the centerline paths of the SVC and PA anatomy of the original Glenn models were left unchanged. The space created by the deflation of the right atrium during the Fontan procedure was accounted for in IVC placement during virtual surgery. In addition, the PA segmentations were enlarged to match the baffle size to avoid creating unrealistic stenoses. Although this is purely a simulation study, all models were constructed under guidance of a surgeon to replicate as closely as possible a realistic surgical implementation of the intended design.

Patient B has a left PA (LPA) stenosis, which is commonly observed in Fontan patients owing to aortic arch override. Three potential Y-graft designs were proposed for this patient model. In Y-graft I, the stenosis is relieved by placing the anastomosis of the left branch at the stenosis. In Y-graft II, the left branch is anastomosed distal to the stenosis, without relieving it. In Y-graft III, the left branch is also anastomosed distal to the stenosis, but the stenosis is relieved. Patient E has heterotaxy and a right PA (RPA) stenosis. Instead of an LPA offset connection, the baffle is anastomosed to the RPA in a mirror image of the other patients, denoted as offset I. The stenosis is relieved in the Y-graft I, T-junction, and offset I designs. An LPA-offset connection without relieving the stenosis (II) is also constructed. We redesigned the Y-graft for patient E, denoted as Y-graft II, by bringing the left branch closer to the SVC-PA junction and less anteriorly convex, based on the simulation results of Y-graft I.

\section{Flow Simulation and Boundary Conditions}

Flow simulations were performed with a stabilized finite element Navier-Stokes solver, ${ }^{19}$ assuming rigid walls and Newtonian flow with a density of $1.06 \mathrm{~g} / \mathrm{cm}^{3}$ and viscosity of $0.04 \mathrm{~g} /(\mathrm{cm} \mathrm{s})$. MeshSim (Simmetrix, Inc, Clifton Park, NY) was used to generate tetrahedral meshes automatically and anisotropic mesh adaptation was performed to ensure mesh convergence. Final meshes consisted of approximately 1 to 1.5 million elements.

During MRI, phase-contrast MRI slices were acquired in the SVC, IVC, LPA, and RPA for each Glenn patient. The SVC waveform from phasecontrast MRI was applied directly to the SVC inflow face by mapping it to a parabolic profile. Because the IVC flow waveforms were acquired in the Glenn patients before surgery, when the IVC was still connected to the right atrium, these waveforms exhibited higher cardiac pulsatility and less respiratory pulsatility compared with typical Fontan patients. ${ }^{20}$ To account for this, the amplitude of the IVC waveform of each Glenn patient was scaled to match typical Fontan data using 4 patients from a previous study, while keeping the mean the same. Additionally, a respiratory model was superimposed on the scaled IVC waveform as done in our previous work. $^{6}$

A 3-element Windkessel circuit model (resistor-capacitorresistor $)^{21-23}$ was applied at each outlet. Predicting changes in pulmonary resistance after Fontan surgery remains an open question. However, inasmuch as this study aims to model immediate postoperative flow conditions, we have assumed that the downstream resistances do not change significantly in the short period after the surgery. Therefore, the same parameters for the Windkessel model were used for the Glenn and Fontan simulations in all patients, so that the comparison of postoperative states among patients remains consistent.

Exercise flow conditions were simulated by increasing IVC flow and decreasing outflow resistances, as done in our previous work. ${ }^{6}$ The mean IVC flow rate was increased by 2 and 3 times $(2 \times, 3 \times)$ to simulate exercise conditions, and the total resistances of each branch was decreased by $5 \%$ and $10 \%$, respectively.

\section{Determination of Performance Parameters}

Mean SVC pressure, wall shear stress (WSS), and power loss were computed with standard methods. ${ }^{7}$ Lagrangian particle tracking ${ }^{15}$ was performed to quantify the hepatic flow distribution in different surgical designs (Figure 2).

It is widely known that the ratio of IVC/SVC flow and LPA/RPA resistances vary widely among patients. Although ideally one would like to 


\section{Glenn $\longrightarrow$ Fontan}

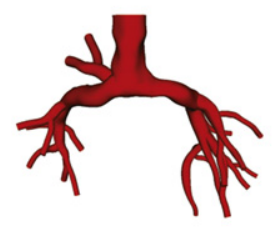

A

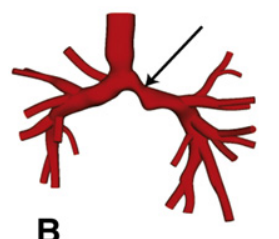

B
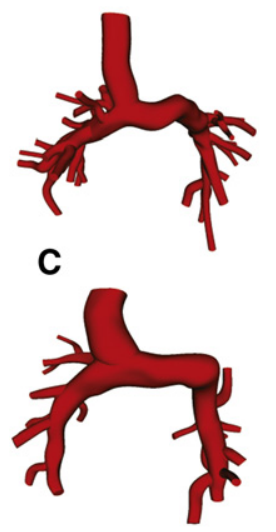

D

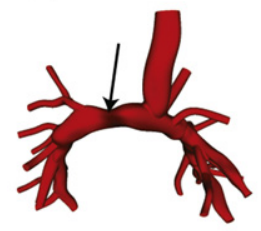

E
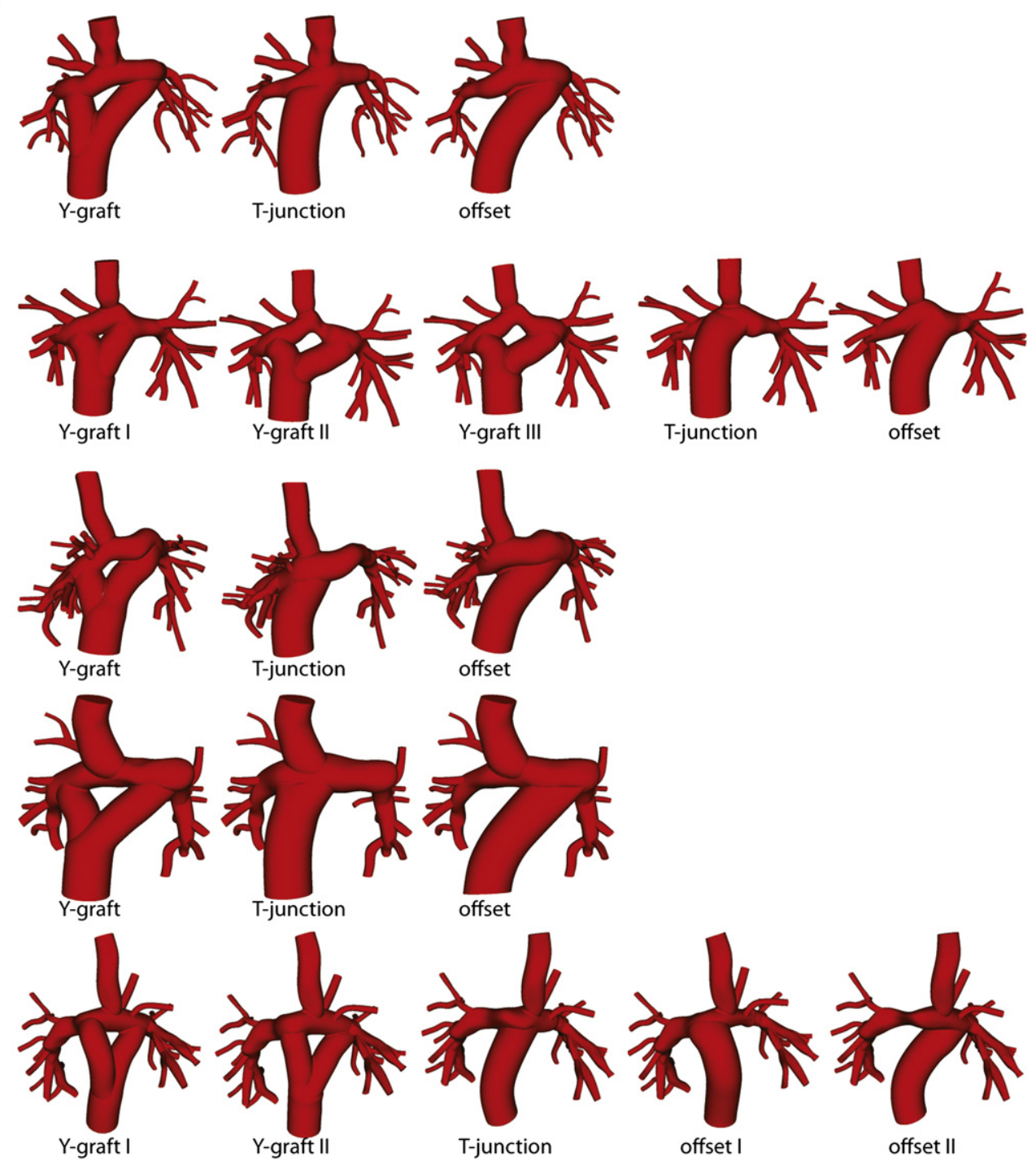

FIGURE 1. Original Glenn models and variations of Fontan geometries for 5 patients. The Y-graft includes a 20-mm trunk and two 15-mm branches. The size of the tube-shaped graft is $20 \mathrm{~mm}$. Patients B and E have a stenosis in the left and right pulmonary arteries, respectively, denoted by arrows.

achieve a 50:50 hepatic flow split for all patients, this will not be possible in every case. Conservation of mass analysis (see Appendix 1) allows us to determine the theoretical optimum for hepatic flow distribution in a given situation.

Table 1 lists the theoretical best hepatic flow distribution for the 5 patients in our study. We observe that a perfect 50:50 split can theoretically exist for 4 of the 5 patients, with patient $\mathrm{A}$ as the exception, for whom the RPA receives more than $80 \%$ of the total inflow.

The main focus of this study is the evaluation of Fontan designs in the immediate postoperative period. However, it is unlikely that pulmonary resistances remain constant over time for most Fontan patients owing to age, growth, and remodeling. We therefore assess the robustness of the hepatic flow distribution to changes in the pulmonary flow split for different surgical designs. For each patient, the Y-graft, offset, and T-junction designs were analyzed under rest conditions for a range of pulmonary resistances to compare the robustness of different designs.

\section{RESULTS}

\section{Hepatic Flow Distribution}

The percentages of hepatic flow to the RPA and LPA, and differences from the theoretical optima at rest, $2 \times$, and $3 \times$ exercise conditions, are shown in Figure 3. In patient A, the Y-graft design is closest to the theoretical optimum, even though patient A's right lung receives more than $80 \%$ of the total venous return. So that we can illustrate how the geometry influences the hemodynamics of hepatic flow distribution, the velocity fields for 3 patients are shown in Figure 4. In the T-junction design of patient A, the SVC jet clearly blocks the hepatic flow entering the LPA and skews it to the RPA. In contrast, the Y-graft design effectively mitigates this effect. 

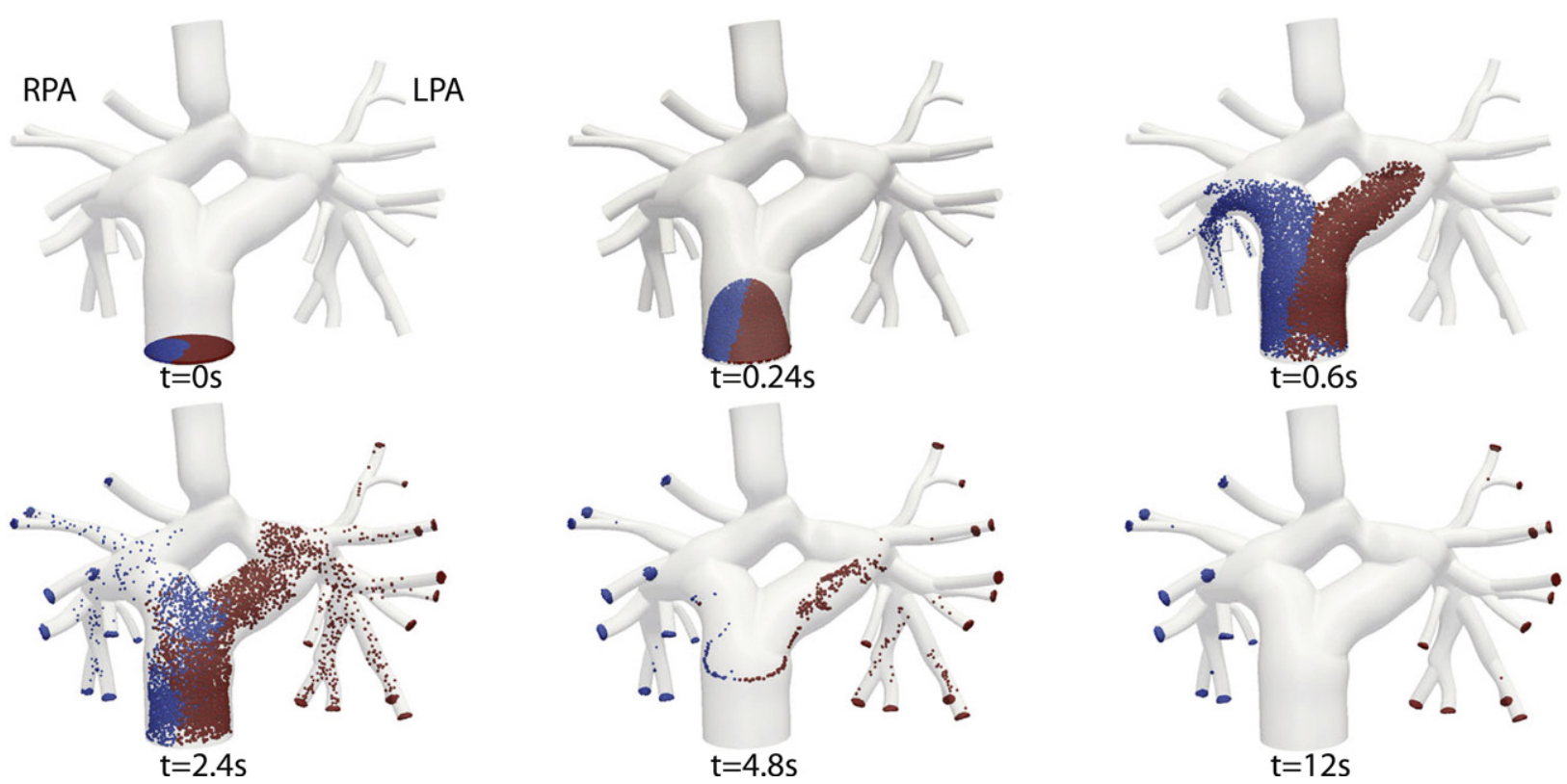

FIGURE 2. Visualization of the particle tracking in the model Y-graft II for patient B. Particle tracking is terminated when particles are washed from the model. $L P A$, Left pulmonary artery; $R P A$, right pulmonary artery.

In patient $\mathrm{B}$, Y-graft I hemodynamics shows that relieving the stenosis with a proximal anastomosis allows the SVC jet to enter the baffle and hinder hepatic flow. However, Y-graft II, which leaves the stenosis intact, achieves a nearly perfect 50:50 distribution at rest. Overall, for patient B, Y-graft II has the best hepatic distribution at rest, whereas the offset design performs best at exercise.

In patient $\mathrm{C}$, the $\mathrm{T}$-junction and offset designs skew hepatic flow to the RPA and LPA, respectively. In both patients $\mathrm{C}$ and $\mathrm{D}$, when the pulmonary flow split is close to 50:50, the offset design has poor performance with a highly skewed hepatic flow split. Overall, for patient C, the Y-graft design has the best hepatic performance, although none of the designs achieves the theoretical optimum.

In patient $\mathrm{D}$, although the $\mathrm{SVC}$ anastomosis is inclined to channel flow to the LPA, Figure 4 shows that the lower section $b c$ of the SVC is ineffective in changing the direction of flow. Improvements are observed in both the Y-graft and T-junction during exercise, and the Y-graft achieves a nearly

TABLE 1. MRI inflow rates, MRI outflow splits, and the TOHFS at rest for the 5 study patients

\begin{tabular}{lcccccc}
\hline Patient & $\begin{array}{c}\text { Age } \\
(\mathbf{y})\end{array}$ & $\begin{array}{c}\text { BSA } \\
\left(\mathbf{m}^{\mathbf{2}}\right)\end{array}$ & $\begin{array}{c}\text { IVC } \\
(\mathbf{m L} / \mathbf{s})\end{array}$ & $\begin{array}{c}\text { SVC } \\
(\mathbf{m L} / \mathbf{s})\end{array}$ & $\begin{array}{c}\text { RPA/LPA } \\
\text { flow split }\end{array}$ & $\begin{array}{c}\text { TOHFS } \\
(\mathbf{R P A} / \mathbf{L P A})\end{array}$ \\
\hline A & 4.8 & 0.63 & 13.4 & 14.4 & $81 / 19$ & $61 / 39$ \\
B & 3.9 & 0.66 & 12.6 & 15.3 & $63 / 37$ & $50 / 50$ \\
C & 2.8 & 0.56 & 6.3 & 15.2 & $54 / 46$ & $50 / 50$ \\
D & 3.0 & 0.61 & 14.8 & 27.8 & $55 / 45$ & $50 / 50$ \\
E & 3.0 & 0.74 & 12.7 & 19.4 & $70 / 30$ & $50 / 50$ \\
\hline
\end{tabular}

MRI, Magnetic resonance imaging; TOHFS, theoretical optimal hepatic flow splits; $B S A$, body surface area; $I V C$, inferior vena cava; $S V C$, superior vena cava; $R P A$, right pulmonary artery; $L P A$, left pulmonary artery.
50:50 split in the $3 \times$ exercise case. Overall, for patient $\mathrm{D}$, the T-junction does best at rest, whereas the Y-graft does best at exercise.

In patient $\mathrm{E}$, the T-junction, offset I, and Y-graft I designs skew hepatic flow strongly to the RPA with a 70:30 (RPA/LPA) pulmonary flow split because of the smooth graft and the low distal RPA resistance. For the T-junction design, hepatic flow distribution remains constant with exercise, whereas Y-graft I progressively improves the distribution from rest to exercise. The skewed hepatic flow is corrected in the Y-graft because the SVC jet suppresses hepatic flow entering the RPA from the right branch (Figure 4). Overall, for patient E, the offset II design results in the best hepatic flow distribution at rest and exercise.

\section{Robustness Test}

The hepatic flow distribution in the Y-graft, offset and T-junction designs for 3 different pulmonary flow splits, and their averaged deviations for a $25 \%$ change in flow split, are shown in Figure 5. The optimal design depends heavily on the pulmonary flow split for individual patients. The Y-graft and T-junction designs are relatively more robust in 2 and 3 patients, respectively. Although the T-junction design underperforms for most patients with the original flow split, deviations in hepatic flow distribution are smaller than those of the offset design.

\section{SVC Pressure}

Pressure levels increase in all models under exercise conditions. The SVC pressure levels in the Y-graft are the same 

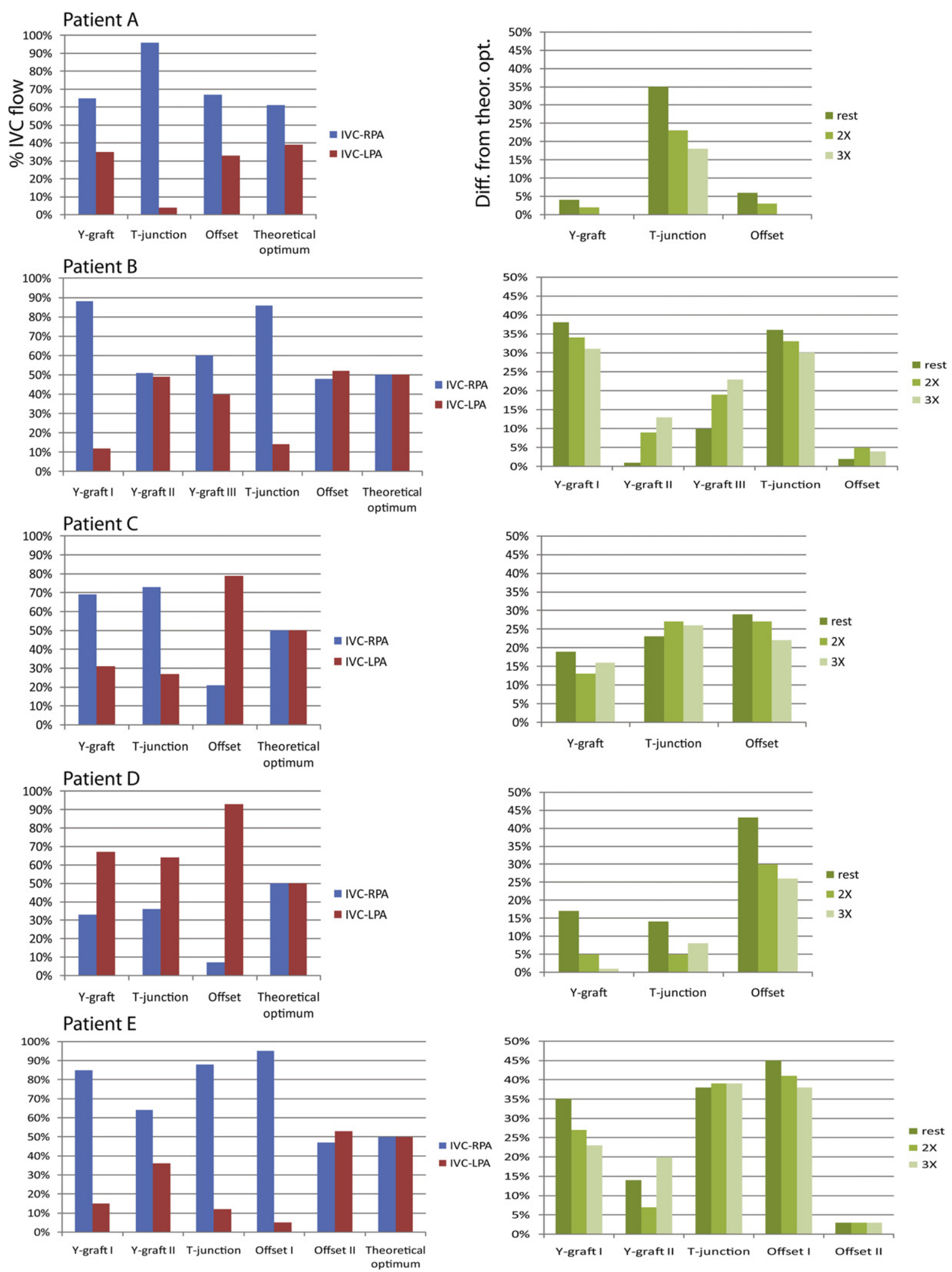

FIGURE 3. Left, Hepatic flow distribution at rest. Right, Differences (percentage of the IVC flow) from the theoretical optima for each design at rest and exercise. Note that the theoretical optima for patient A at rest, $2 \times$, and $3 \times$, are 61/39,70/30 and 72/28, respectively, and that a 50:50 split cannot be achieved in theory. $I V C$, Inferior vena cava; $R P A$, right pulmonary artery; $L P A$, left pulmonary artery. 

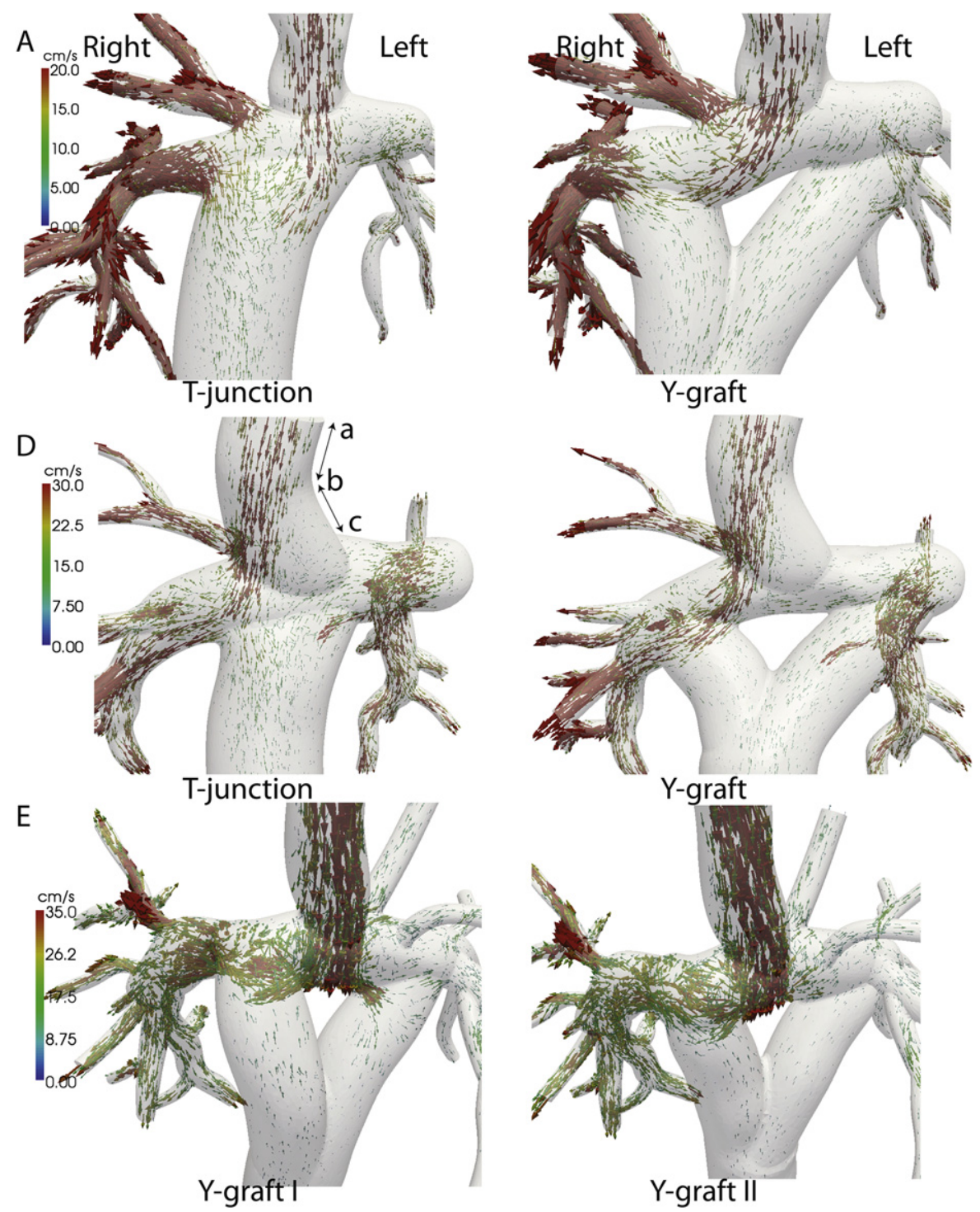

FIGURE 4. Time-averaged velocity vectors in the Y-graft and T-junction models for patients A, D, and E. In the T-junction design for patient A, the SVC jet blocks the hepatic flow entering the LPA. In patient D, most SVC flow is directed to the RPA owing to a curved SVC. In patient E, Y-graft II improves the hepatic flow distribution by having a straight proximal branch for the RPA, in which the SVC jet blocks hepatic flow going to the RPA from the right branch, compared with Y-graft I. SVC, Superior vena cava; $L P A$, left pulmonary artery; RPA, right pulmonary artery.

or slightly lower than those in the T-junction and offset designs at rest and exercise conditions in 5 patients (Table 2). This finding agrees with our previous work, which identified a pressure shielding effect causing lower SVC pressure with the Y-graft design owing to the lack of a head-on flow collision in the junction. ${ }^{7}$

\section{Power Loss}

Table 2 gives the power loss values for the Y-graft, T-junction, and offset designs for each patient. Energy loss increases with increasing exercise levels. The T-junction designs result in the highest energy losses in most patients owing to a direct flow collision, agreeing with previous studies.

\section{WSS}

Mean WSS values on the IVC graft generally increase with increasing inflow rates (Table 2). Exceptions to this are the T-junction and offset designs for patient $\mathrm{C}$, in whom the SVC jet enters the tube-shaped graft and increases the WSS at a low IVC flow rate. The Y-graft and offset designs result in lower mean WSS values on the graft than the T-junction design. This is consistent with the fact that the T-junction design usually causes more energy 

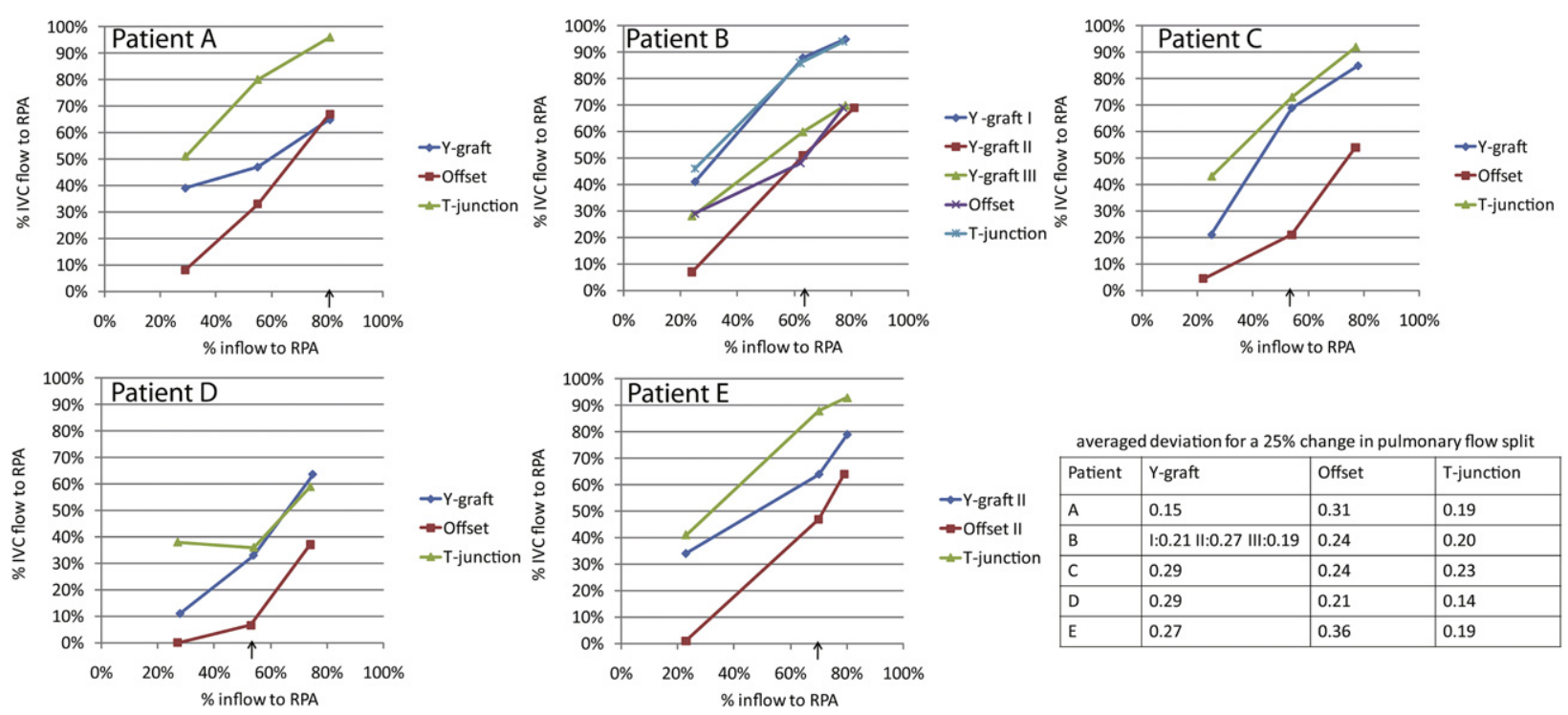

FIGURE 5. Hepatic flow distribution changes with variations in pulmonary flow split. Patients' original pulmonary flow splits are marked by the arrows at the $x$-axis. The table shows the averaged deviations with respect to the original hepatic flow distribution for a $25 \%$ change in pulmonary flow split. IVC, Inferior vena cava; $R P A$, right pulmonary artery.

dissipation. Time-averaged WSS at rest for 2 representative patients is shown in Figure 6. The other 3 patients exhibited similar behavior.

\section{Averaged Results}

By averaging the hepatic distributions and power losses in the best performing Y-graft, T-junction, and offset designs over the 5 patients in the study (see Figure 7), we found that the Y-graft design has the lowest average difference from the theoretical optima under rest and exercise conditions (ie, the Y-graft design results in more even hepatic flow distribution than other designs overall) and that the distribution approaches the theoretical optima with increasing exercise level. Significant differences $(P<.05)$

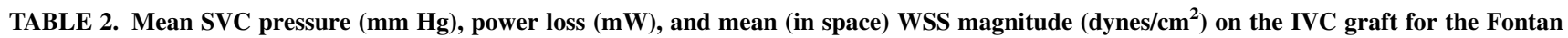
models. Compared with the best Y-graft design for the same patient, increases in power loss for the T-junction and offset designs are also shown

\begin{tabular}{|c|c|c|c|c|c|c|c|c|c|c|}
\hline \multirow[b]{2}{*}{ Patient } & \multirow[b]{2}{*}{ Model } & \multicolumn{3}{|c|}{ SVC pressure } & \multicolumn{3}{|c|}{ Power loss } & \multicolumn{3}{|c|}{ Mean WSS } \\
\hline & & Rest & $2 \times$ & $3 \times$ & Rest & $2 \times$ & $3 \times$ & Rest & $2 \times$ & $3 \times$ \\
\hline \multirow[t]{3}{*}{ A } & Y-graft & 15.3 & 19.4 & 22.9 & 5.1 & 14.5 & 24.9 & 2.3 & 4.6 & 7.2 \\
\hline & T-junction & 15.5 & 19.6 & 23.4 & $16 \%$ & $14 \%$ & $14 \%$ & 4.5 & 5.9 & 7.9 \\
\hline & Offset & 15.4 & 19.7 & 23.4 & $10 \%$ & $15 \%$ & $17 \%$ & 1.9 & 4.5 & 7.4 \\
\hline \multirow[t]{5}{*}{ B } & Y-graft I & 12.9 & 16.7 & 19.9 & 2.4 & 5.4 & 8.6 & 6.5 & 6.5 & 8.7 \\
\hline & Y-graft II & 12.8 & 16.6 & 19.8 & 2.0 & 4.6 & 7.5 & 2.2 & 5.2 & 7.6 \\
\hline & Y-graft III & 12.8 & 16.6 & 19.8 & 2.0 & 4.7 & 7.6 & 2.3 & 4.6 & 7.0 \\
\hline & T-junction & 13.1 & 17.1 & 20.5 & $60 \%$ & $70 \%$ & $76 \%$ & 6.1 & 6.8 & 9.0 \\
\hline & Offset & 13.0 & 17.0 & 20.4 & $40 \%$ & $57 \%$ & $64 \%$ & 3.1 & 5.1 & 7.4 \\
\hline \multirow[t]{3}{*}{$\mathrm{C}$} & Y-graft & 10.4 & 11.9 & 13.0 & 1.8 & 2.9 & 4.1 & 3.0 & 3.3 & 4.2 \\
\hline & T-junction & 10.4 & 11.9 & 13.0 & $0 \%$ & $7 \%$ & $5 \%$ & 5.5 & 5.4 & 5.3 \\
\hline & Offset & 10.4 & 11.9 & 13.1 & $0 \%$ & $10 \%$ & $15 \%$ & 3.0 & 2.2 & 2.4 \\
\hline \multirow[t]{3}{*}{ D } & Y-graft & 10.8 & 13.5 & 15.8 & 5.8 & 12.8 & 19.9 & 3.8 & 6.9 & 9.6 \\
\hline & T-junction & 10.9 & 13.7 & 16.1 & $10 \%$ & $15 \%$ & $17 \%$ & 4.0 & 4.5 & 5.9 \\
\hline & Offset & 10.8 & 13.6 & 16.0 & $5 \%$ & $11 \%$ & $16 \%$ & 2.5 & 6.0 & 9.8 \\
\hline \multirow[t]{5}{*}{ E } & Y-graft I & 22.9 & 27.7 & 31.7 & 5.14 & 10.8 & 17.1 & 3.9 & 5.7 & 8.0 \\
\hline & Y-graft II & 22.8 & 27.6 & 31.7 & 4.7 & 9.7 & 15.6 & 2.9 & 4.7 & 7.15 \\
\hline & T-junction & 22.9 & 27.8 & 31.8 & $14 \%$ & $15 \%$ & $15 \%$ & 5.4 & 7.1 & 10.2 \\
\hline & Offset I & 22.8 & 27.6 & 31.6 & $-1 \%$ & $-2 \%$ & $-5 \%$ & 3.1 & 6.6 & 6.8 \\
\hline & Offset II & 23.4 & 28.6 & 33.1 & $65 \%$ & $86 \%$ & $90 \%$ & 2.7 & 4.6 & 8.1 \\
\hline
\end{tabular}

SVC, Superior vena cava; WSS, wall shear stress; IVC, inferior vena cava. 

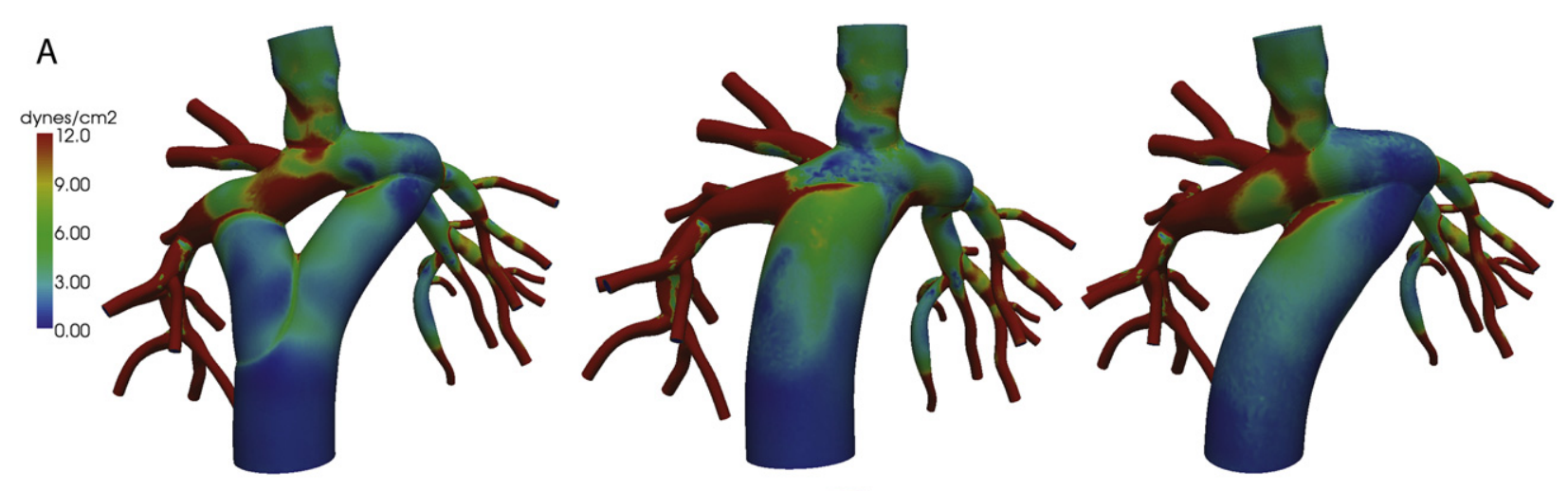

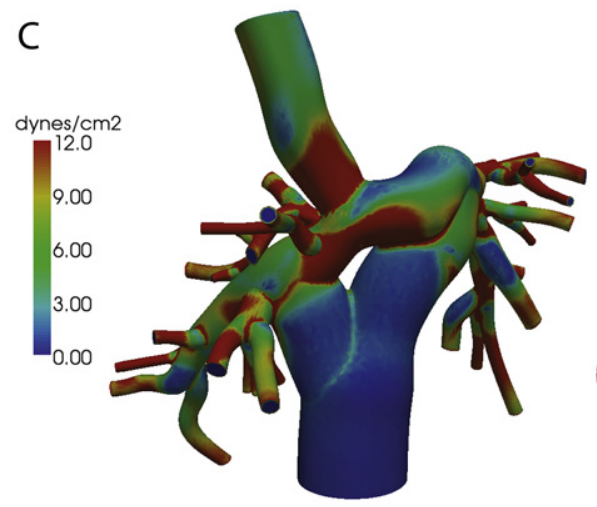

Y-graft

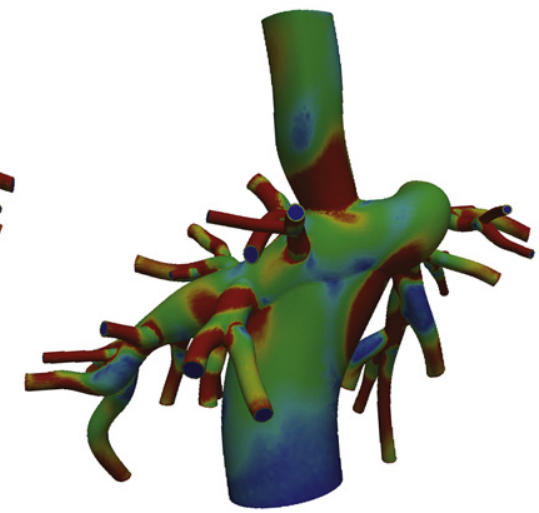

T-junction

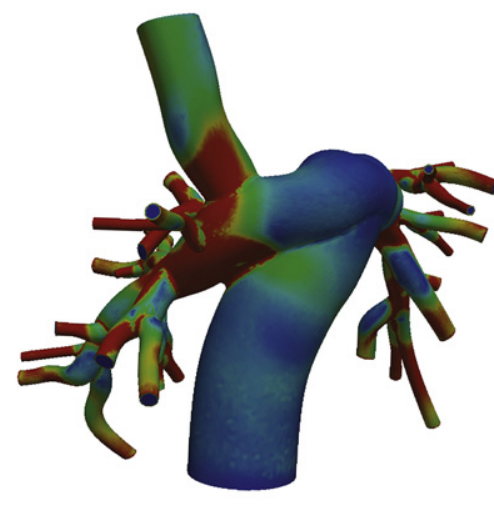

Offset

FIGURE 6. Contours of time-averaged wall shear stress $\left(\right.$ dynes $\left./ \mathrm{cm}^{2}\right)$ at rest for patients A and C.

are found in the mean hepatic flow distribution and power loss between the Y-graft and T-junction designs.

\section{DISCUSSION}

In this study, a multiparameter approach was used to evaluate 3 Fontan designs in 5 patients, with particular focus on hepatic flow distribution. This multiple-patient series has demonstrated that the Y-graft design can significantly improve hepatic flow distribution and moderately improve energy loss and SVC pressure. However, our results emphasize that no one-size-fits-all design achieves satisfactory hepatic distributions in all patients. The hemodynamics affecting flow distribution are nonintuitive in many cases, and small differences in geometry can dramatically influence results. Because these subtle changes are not easily elucidated with standard imaging modalities, simulations should be used to determine the best candidates for a Y-graft preoperatively and to refine the graft design for each patient.

\section{Hepatic Flow Distribution}

Hepatic flow distribution in the Fontan is driven by multiple factors. The SVC jet can prevent hepatic flow from reaching the LPA, and this was the leading cause of uneven hepatic flow distribution in our study, agreeing with previous results of Dasi and associates. ${ }^{16}$ Although
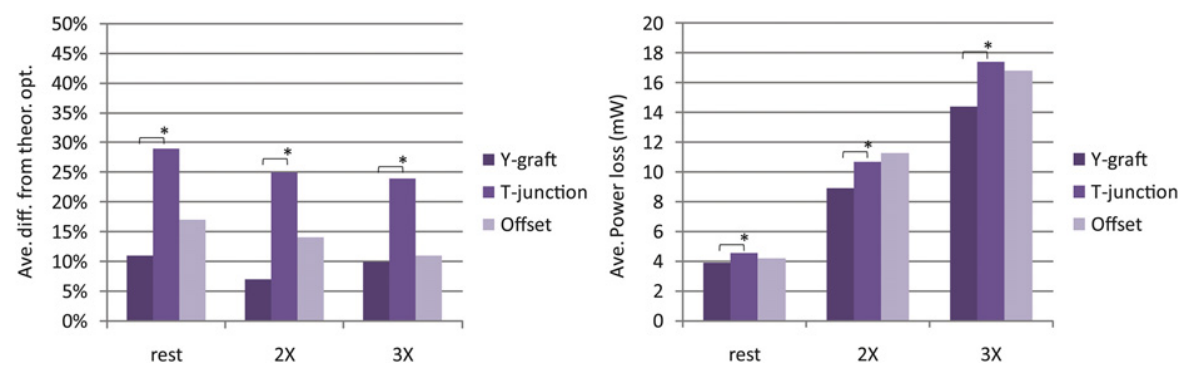

FIGURE 7. Averaged differences from the theoretical optima and power losses over 5 patients. The best performing of the Y-graft and offset designs for patients $\mathrm{B}$ and $\mathrm{E}$ are used. The differences between the Y-graft and T-junction designs are statistically significant $\left({ }^{*} P<.05\right)$. 
previous studies in idealized geometries showed that the T-junction design effectively mixes the IVC and SVC flows and distributes the hepatic flow evenly, ${ }^{13}$ these phenomena were not observed uniformly in the patientspecific geometries in our study. The Y-graft design distributes hepatic flow more evenly than the T-junction in most patients, but the wrong choice of Y-graft may lead to unfavorable hemodynamics. The LPA-offset design generally achieves satisfactory hepatic distribution in patients with high LPA resistance but an unfavorable distribution in patients with an equal pulmonary flow split. This result is consistent with previous work of Bove and colleagues, ${ }^{14}$ which shows that the IVC flow in the total cavopulmonary connection and traditional extracardiac Fontan models is skewed to the LPA with a total pulmonary flow split close to 50:50.

The nonintuitive result for patient $\mathrm{D}$ demonstrates that the geometry of the SVC and relevant boundary conditions plays an important role in distributing the IVC and SVC flows. The hepatic flow distribution depends on the SVC flow distribution (equation 2) with an inverse relationship between the percentages of hepatic and SVC flow going to the RPA. Thus the effect of the SVC-PA anastomosis on the hepatic flow distribution should be carefully considered in surgical planning for the Glenn procedure.

Although the maximum change in hepatic flow distribution from rest to exercise was less than $20 \%$, values generally approached their theoretical optima during exercise. When the IVC flow rate is increased during exercise, the interaction between the 2 caval flows in the T-junction design generally enhances mixing and distributes hepatic flow more evenly. Salim and associate ${ }^{24}$ showed that the contribution of IVC flow to the cardiac output increases from $45 \%$ at 2.5 to 3 years old to $65 \%$ (the adult value) at 6.6 years old. In our study, $45 \%$ to $48 \%$ of systemic venous return is contributed by the IVC in 2 patients (mean 4.3 years), whereas the IVC contribution is between $29 \%$ and $35 \%$ in 3 patients (mean 2.9 years). Thus, simulations under exercise conditions may reveal some trends in the hepatic flow distribution with increasing age, although changes in distribution of caval flow to the PAs with age are still unknown. ${ }^{25}$

Our robustness test confirms that hepatic flow distribution depends strongly on the pulmonary flow split. For some patients, the Y-graft design is relatively more robust than the offset design for a wide range of flow conditions with less chance to skew all IVC flow to one lung, but this characteristic is not universal for all Y-graft designs. Although the T-junction design skews hepatic flow with the patients' original pulmonary flow splits, it performs better under the conditions with a high RPA resistance owing to a slight RPA-offset for the anastomosis. Thus, the optimal working condition for the LPA-offset design is opposite. No design emerged as the clear winner over a wide range of flow splits.

\section{Power Loss}

Compared with traditional designs, the Y-graft design reduces energy dissipation by bifurcating the IVC to decrease flow competition. The Y-graft design reduces energy loss at rest by $5 \%$ to $27 \%$ in 4 of 5 patients compared with the offset design. The differences in energy loss are more pronounced during exercise, agreeing with previous work.

\section{SVC Pressure}

It is clinically widely accepted that lower Fontan pressure generally correlates with better outcomes. Overall, the Y-graft design offers moderate reductions in the SVC pressure compared with the T-junction and offset designs. In the T-junction design, a higher SVC pressure is usually required to overcome competing flow from the IVC. However, the average SVC pressure differences are not as pronounced as those reported in our previous work, ${ }^{7}$ likely because IVC flow rates were lower in the younger patients in this study. This trend suggests that the significance of the pressure shielding effect observed with the Y-graft may increase as patient's age and their relative IVC flow increases.

\section{WSS}

The Y-shaped grafts have lower mean WSS values than the tube-shaped grafts. These results are qualitatively consistent with our previous work on idealized Y-graft shape optimization, ${ }^{8}$ which shows a trade-off between energy efficiency and areas of low WSS. Whereas the WSS values in the Y-graft are generally comparable with values in offset designs, further investigation into this issue is warranted, and in our current clinical initial implementation of this surgical modification, we have chosen to exclude patients with known thrombotic tendency. The impingement of SVC flow results in a relatively high WSS region in the intervening segment of the PA (Figure 6), which does not suggest an increased likelihood of flow stasis and thrombus formation in that region.

\section{Ranking}

On the basis of power loss and hepatic flow distribution, the proposed surgical designs are ranked for each patient (Table 3) at rest and exercise. For energy loss, the Y-graft design is superior to the others in 4 of 5 patients regardless of rest or exercise conditions, and offset $I$ is superior in patient E. However, more variations emerge in the ranking on the basis of the hepatic flow distribution.

For patients $\mathrm{A}$ and $\mathrm{C}$, the Y-graft design is clearly the final winner, inasmuch as it was uniformly ranked first in both energy loss and hepatic flow distribution. For patient B, the offset design provides $10 \%$ improvement in the hepatic flow distribution at exercise, with better robustness to the pulmonary flow split, but produces over $40 \%$ more power loss than Y-graft II. We therefore select Y-graft II as the final candidate for patient B. For this patient, we note that Y-graft III 
TABLE 3. Ranking of energy loss and hepatic flow distribution for each patient

\begin{tabular}{|c|c|c|c|c|c|c|c|c|}
\hline \multirow[b]{2}{*}{ Patient } & \multirow[b]{2}{*}{ Model } & \multicolumn{3}{|c|}{ Power loss } & \multicolumn{3}{|c|}{ Hepatic flow } & \multirow{2}{*}{$\begin{array}{c}\text { Recommended } \\
\text { designs }\end{array}$} \\
\hline & & Rest & $2 \times$ & $3 \times$ & Rest & $2 \times$ & $3 \times$ & \\
\hline \multirow[t]{3}{*}{ A } & Y-graft & 1 & 1 & 1 & 1 & 1 & 1 & $\nu$ \\
\hline & T-junction & 3 & 2 & 2 & 3 & 3 & 3 & \\
\hline & Offset & 2 & 3 & 3 & 2 & 2 & 2 & \\
\hline \multirow[t]{5}{*}{ B } & Y-graft I & 3 & 3 & 3 & 5 & 5 & 5 & \\
\hline & Y-graft II & 1 & 1 & 1 & 1 & 2 & 2 & 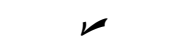 \\
\hline & Y-graft III & 2 & 2 & 2 & 3 & 3 & 3 & \\
\hline & T-junction & 5 & 5 & 5 & 4 & 4 & 4 & \\
\hline & Offset & 4 & 4 & 4 & 2 & 1 & 1 & \\
\hline \multirow[t]{3}{*}{$\mathrm{C}$} & Y-graft & 1 & 1 & 1 & 1 & 1 & 1 & 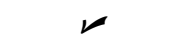 \\
\hline & T-junction & 3 & 2 & 2 & 2 & 2 & 3 & \\
\hline & Offset & 2 & 3 & 3 & 3 & 2 & 2 & \\
\hline \multirow[t]{3}{*}{ D } & Y-graft & 1 & 1 & 1 & 2 & 1 & 1 & 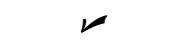 \\
\hline & T-junction & 3 & 3 & 3 & 1 & 1 & 2 & \\
\hline & Offset & 2 & 2 & 2 & 3 & 3 & 3 & \\
\hline \multirow[t]{5}{*}{$\mathrm{E}$} & Y-graft I & 3 & 3 & 3 & 3 & 3 & 3 & \\
\hline & Y-graft II & 2 & 2 & 2 & 2 & 2 & 2 & $\nu$ \\
\hline & T-junction & 4 & 4 & 4 & 4 & 4 & 5 & \\
\hline & Offset I & 1 & 1 & 1 & 5 & 5 & 4 & \\
\hline & Offset II & 5 & 5 & 5 & 1 & 1 & 1 & \\
\hline
\end{tabular}

The ranking of the hepatic flow distribution is based on the differences from the theoretical optima. In patients $\mathrm{C}$ and $\mathrm{D}$, there are two designs tied for the hepatic flow distribution.

overcomes the disadvantage of Y-graft II in robustness, while keeping the power loss almost unchanged. If we were to weight the robustness more heavily, then Y-graft III would likely be the recommended choice. For patient $\mathrm{D}$, the Y-graft design is chosen because of lower energy losses and progressive improvements in the hepatic flow distribution during exercise, but the T-junction would be preferred if the robustness were weighted more heavily than the power loss. For patient E, Y-graft II is chosen because it is well balanced between energy loss and the hepatic flow distribution, and it is more robust to changes in the pulmonary flow split compared with other designs. These surgical recommendations are limited to the patients we studied. Generalization of these choices should be made carefully, inasmuch as metrics used in this study could change as additional clinical data are obtained in future work. The reader should be cautioned that not all variants of the Y-graft design that we tested in this study performed well. However, the potential of the Y-graft design to improve hemodynamic performance is promising, and a variant of the Y-graft design was our final recommendation for all patients.

\section{Technical Feasibility}

In this work we virtually implanted a $20 \times 15 \times 15$ Y-graft in each patient, but it should be noted that this configuration is not currently commercially available. Our proposed design therefore requires customized graft construction for each patient using commercially available sections of conduit. Space constraints may present a surgical challenge for the PA anastomoses. Inasmuch as offsets to both the LPA and RPA with a 20-mm graft are routinely performed already, this should indicate available space to offset to either side of the SVC anastomosis using a Y-graft design. Of course, technical feasibility will be demonstrated only through clinical implementation.

\section{LIMITATIONS}

A main limitation in this study is the lack of postoperative data on patients' resistances and caval flow rates. Although this information would not typically be available in a clinical preoperative design study, the development of sophisticated models that can predict changes and remodeling in inflows and outlet boundary conditions will be crucial for future surgical design and management of patients. The evolution of this dynamic process is still an open question and is beyond the scope of this study. Validation studies of preoperative design, long-term hemodynamics, and uncertainty analysis should be incorporated into future work.

In this study, we assumed an optimal hepatic flow distribution of 50:50 and evaluated designs according to how closely they met this criterion. The target values chosen in this study could be adjusted on an individual basis as our understanding of the relationship between hepatic flow concentration and lung development is improved. Because the theoretical optimum was not achieved for all patients in the study, it is possible that further design optimization may improve some underperforming Y-grafts. Future work using patient-specific optimization would likely lead to further design refinement in some cases, inasmuch as this would allow for more systematic exploration of the design space. In addition, Bove and colleagues ${ }^{14}$ show lateraltunnel Fontan models constructed from hemi-Fontan models result in even hepatic flow distribution and lower power loss because of better mixing in the right atrium, so future studies on the Y-graft should include comparisons with the lateral-tunnel Fontan as well. Finally, inevitable discrepancies will occur between the virtual design and its actual surgical implementation. This introduces certain geometric uncertainties that could result in differences between computer simulations and the actual conditions. In addition, the use of rigid walls and the Newtonian assumption for blood may affect the results presented in this study.

\section{CONCLUSIONS}

Using 5 Glenn patient-specific models, we performed virtual Fontan operations and compared the hemodynamic performance of the Y-graft, T-junction, and offset configurations. We have demonstrated that the geometry considerably influences the hepatic flow distribution, and the hepatic flow split is not necessarily equal to the pulmonary flow split. Theoretical analysis showed that a 50:50 hepatic flow split is not attainable for some patients. Overall, the 
Y-graft design results in more even hepatic flow distribution and moderate improvements in energy loss and SVC pressure. The offset design is able to achieve an even hepatic flow distribution for patients with highly unequal pulmonary flow splits, but it is sensitive to variations in pulmonary flow split. The results of this study indicate that graft designs should be optimized for individual patients before surgery. In conclusion, the Y-graft is a promising new design that warrants testing in clinical application and long-term clinical trials. With further validation, simulations should be used to identify the best candidates for the Y-graft procedure and to rule out those patients who should continue to receive conventional treatments.

We are grateful to Shawn Shadden for sharing his expertise and codes in particle tracking as well as Sethuraman Sankaran, Frandics Chan, Heidi Terwey, and Mary Hunt Martin for their helpful discussions and expertise. We also wish to acknowledge the use of Simvascular (simtk.org), as well as the numerical modeling expertise of Nathan Wilson and Charles Taylor.

\section{References}

1. Migliavacca F, Dubini G, Bove EL, de Leval MR. Computational fluid dynamics simulations in realistic 3-D geometries of the total cavopulmonary anastomosis: the influence of the inferior caval anastomosis. J Biomech Eng. 2003;125:805-13.

2. Vignon-Clementel IE, Marsden AL, Feinstein JA. A primer on computational simulation in congenital heart disease for the clinician. Progr Pediatr Cardiol. 2010;30:3-13

3. de Zelicourt DA, Marsden AL, Fogel MA, Yoganathan AP. Imaging and patientspecific simulations for the Fontan surgery: current methodologies and clinical applications. Progr Pediatr Cardiol. 2010;30:31-44.

4. Marsden AL, Reddy VM, Shadden SC, Chan FP, Taylor CA, Feinstein JA. A new multi-parameter approach to computational simulation for Fontan assessment and redesign. Congenit Heart Dis. 2010;5:104-17.

5. de Leval MR, Dubini G, Migliavacca F, Jalali H, Camporini G, Redington A, et al. Use of computational fluid dynamics in the design of surgical procedures: application to the study of competitive flows in cavo-pulmonary connections. $J$ Thorac Cardiovasc Surg. 1996;111:502-13.

6. Marsden AL, Vignon-Clementel IE, Chan F, Feinstein JA, Taylor CA. Effects of exercise and respiration on hemodynamic efficiency in CFD simulations of the total cavopulmonary connection. Ann Biomed Eng. 2007;35:250-63.

7. Marsden AL, Bernstein AJ, Reddy VM, Shadden S, Spilker RL, Chan FP, et al. Evaluation of a novel Y-shaped extracardiac Fontan baffle using computational fluid dynamics. J Thorac Cardiovasc Surg. 2009;137:394-403.

8. Yang W, Feinstein JA, Marsden AL. Constrained optimization of an idealized Yshaped baffle for the Fontan surgery at rest and exercise. Comput Meth Appl Mech Engrg. 2010;199:2135-49.

9. Bazilevs Y, Hsu MC, Besnon DJ, Sankaran S, Marsden AL. Computational fluidstructure interaction: methods and application to a total cavopulmonary connection. Comput Mech. 2008;45:77-89.

10. Soerensen DD, Pekkan K, de Zelicourt D, Sharma S, Kanter K, Fogel M, et al. Introduction of a new optimized total cavopulmonary connection. Ann Thorac Surg. 2007;83:2182-90

11. Marino BS. Outcomes after the Fontan procedure. Curr Opin Pediatr. 2002;14: 620-6.

12. Pike NA, Vricella LA, Feinstein JA, Black MD, Reitz BA. Regression of severe pulmonary arteriovenous malformations after Fontan revision and hepatic factor rerouting. Ann Thorac Surg. 2004;78:697-9.

13. Walker PG, Oweis GF, Watterson KG. Distribution of hepatic venous blood in the total cavo pulmonary connection: an in vitro study into the effects of connection geometry. J Biomech Eng. 2001;123:558-64.

14. Bove EL, de Leval MR, Migliavacca F, Guadagni G, Dubini G. Computational fluid dynamics in the evaluation of hemodynamic performance of cavopulmonary connections after the Norwood procedure for hypoplastic left heart syndrome. J Thorac Cardiovasc Surg. 2003;126:1040-7.
15. Shadden SC, Taylor CA. Characterization of coherent structures in the cardiovascular system. Ann Biomed Eng. 2008;36:1152-62.

16. Dasi LP, Whitehead K, Pekkan K, de Zelicourt D, Katajima H, Sundareswaran K, et al. Pulmonary hepatic flow distribution in total cavopulmonary connections: extracardiac versus intracardiac. J Thorac Cardiovasc Surg. 2011;141:207-14.

17. Troianowski G, Taylor CA, Feinstein JA, Vignon-Clementel I. Threedimensional simulations in Glenn patients: clinically based boundary conditions, hemodynamic results and sensitivity to input data. J Biomech Eng. In press.

18. Schmidt JP, Delp SL, Sherman MA, Taylor CA, Pande VS, Altman RB. The Simbios National Center: systems biology in motion. Proc IEEE Inst Electr Electron Eng. 2008;96:1266-80.

19. Taylor CA, Hughes TJR, Zarins CK. Finite element modeling of blood flow in arteries. Comput Method Appl Mech Engrg. 1998;158:155-96.

20. Hsia T, Khambadkone S, Redington AN, Migliavacca F, Deanfield JE, de Leval MR. Effects of respiration and gravity on infradiaphragmatic venous flow in normal and Fontan patients. Circulation. 2000;102(19 suppl III):III148-53.

21. Vignon-Clementel IE, Figueroa CA, Jansen KE, Taylor CA. Outflow boundary conditions for three-dimensional finite element modeling of blood flow and pressure in arteries. Comput Meth Appl Mech Engrg. 2006;195:3776-96.

22. Spilker RL, Feinstein JA, Parker DW, Reddy VM, Taylor CA. Morphometrybased impedance boundary conditions for patient-specific modeling of blood flow in pulmonary arteries. Ann Biomed Eng. 2007;35:546-9.

23. Vignon-Clementel IE, Figueroa CA, Jansen KE, Taylor CA. Outflow boundary conditions for 3D simulations of non-periodic blood flow and pressure fields in deformable arteries. Comput Meth Biomech Biomed Engrg. 2010;13:625-40.

24. Salim MA, DiSessa TG, Arheart KL, Alpert BS. Contribution of superior vena caval flow to total cardiac output in children: a Doppler echocardiographic study. Circulation. 1995;92:1860-5

25. Fogel MA, Weinberg PM, Rychik J, Hubbard A, Jacobs M, Spray TL, et al. Caval contribution to flow in the branch pulmonary arteries of Fontan patients with a novel application of magnetic resonance presaturation pulse. Circulation. 1999;99:1215-21.

\section{APPENDIX 1}

\section{Theoretical Optimum for Hepatic Flow Distribution}

We assume that hepatic flow is well mixed in the IVC such that the hepatic and IVC flow distributions are the same and the theoretical optimum for the hepatic flow distribution is the split closest to 50:50 that satisfies conservation of mass.

Let us derive the dependence of the hepatic flow distribution on the vena caval and pulmonary distributions (see Figure 8 for a schematic representation of the Fontan

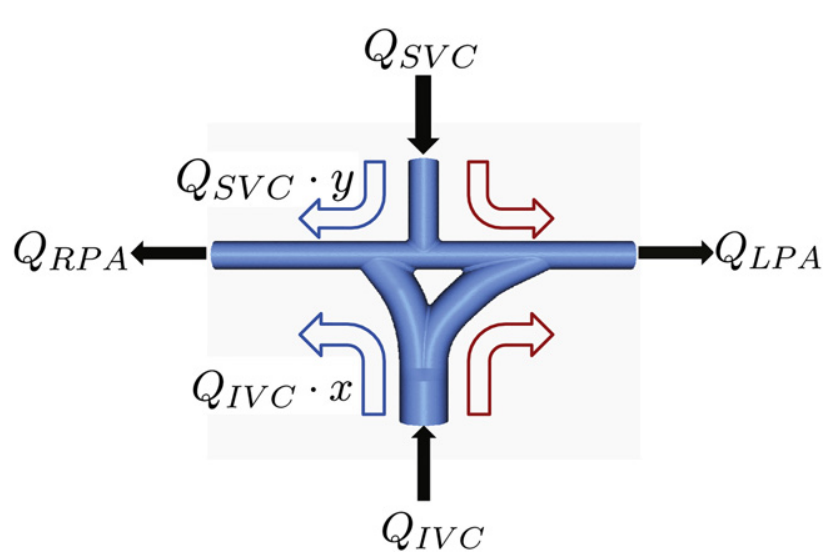

FIGURE 8. Based on conservation of mass, we have $Q_{R P A}=Q_{I V C} \cdot x+$ $Q_{S V C} \cdot y$ and $Q_{L P A}=Q_{I V C} \cdot(1-x)+Q_{S V C} \cdot(1-y)$, where $x$ is the fraction of hepatic flow going to the RPA and $y$ is the fraction of SVC flow going to the RPA. $S V C$, Superior vena cava; $I V C$, inferior vena cava; $R P A$, right pulmonary artery; $L P A$, left pulmonary artery; $Q$, flow rate. 
configuration). Basic conservation of mass dictates that the ratio between the RPA flow and the LPA flow can be defined as

$$
\frac{Q_{R P A}}{Q_{L P A}}=\frac{Q_{I V C} \cdot x+Q_{S V C} \cdot y}{Q_{I V C} \cdot(1-x)+Q_{S V C} \cdot(1-y)}=\frac{f_{s}}{1-f_{s}},
$$

where $Q$ is the flow rate, $f_{s}$ is the fraction of total inflow going to the RPA, $x$ is the fraction of hepatic flow going to the RPA, and $y$ is the fraction of SVC flow going to the RPA. Then, the hepatic flow distribution can be expressed by

$$
x=f_{s}+\frac{Q_{S V C}}{Q_{I V C}} \cdot\left(f_{s}-y\right) .
$$

Equation 2 shows that the hepatic flow distribution is a function of the inflow rates, outflow split, and percentage of SVC flow going to the RPA.

For a given hepatic flow split $x$, the bounds of the RPA flow are

$$
Q_{I V C} \cdot x \leq Q_{R P A} \leq Q_{I V C} \cdot x+Q_{S V C} .
$$

Thus, a 50:50 hepatic flow split cannot exist when $Q_{R P A}<Q_{I V C} \cdot 0.5$ or $Q_{R P A}>Q_{I V C} \cdot 0.5+Q_{S V C}$. Since $Q_{R P A}$ is given as boundary condition, when a 50:50 split is infeasible, the theoretical optimum for the hepatic flow distribution is defined as the value closest to 50:50 achieved by taking $y=0$ or $y=1$, ie, $Q_{I V C} \cdot x=Q_{R P A}$ or $Q_{I V C} \cdot x+Q_{S V C}=Q_{R P A}$ 